\title{
A Novel Nanotube-Based Fiber Laser for Ultrashort Pulse Generation and Fast Measurements
}

\author{
Jie Zhan1,2, Jun Qin ${ }^{2 *}$, Siwei Tan1, Shugang Liu1, Renlong Zhou ${ }^{1}$, Ying Chen² \\ ${ }^{1}$ College of Physics and Electronic Science, Hunan University of Science and Technology, Xiangtan, China \\ ${ }^{2}$ Department of Electrical and Computer Engineering, Southern Illinois University, Carbondale, IL, USA \\ Email: *jqin@siu.edu
}

How to cite this paper: Zhan, J., Qin, J., Tan, S.W., Liu, S.G., Zhou, R.L. and Chen, Y. (2018) A Novel Nanotube-Based Fiber Laser for Ultrashort Pulse Generation and Fast Measurements. Modern Instrumentation, 7, 24-34.

https://doi.org/10.4236/mi.2018.72003

Received: December 28, 2017

Accepted: April 7, 2018

Published: April 10, 2018

Copyright $\odot 2018$ by authors and Scientific Research Publishing Inc. This work is licensed under the Creative Commons Attribution International License (CC BY 4.0).

http://creativecommons.org/licenses/by/4.0/

\section{Open Access}

\begin{abstract}
We propose a nanotube-based erbium-doped fiber laser that can deliver conventional soliton (CS) and stretched pulse (SP) based on D-shaped fiber saturable absorber (DF-SA) where evanescent-field interaction works. The novel Nanotube-based Fiber Laser can generate SP or CS by tuning pump power and polarization controller (PC) properly. The net cavity dispersion of laser is slightly negative. In our experiment, by optimizing the PC in the cavity, CS and SP can be obtained at the central wavelengths of $1530.6 \mathrm{~nm}$ and 1530.3 $\mathrm{nm}$ due to on carbon nanotubes and the spectral filtering effect induced by nonlinear polarization rotation. Although the acquired CS and SP nearly have the same central wavelengths, they show distinct optical spectra, 3-dB bandwidths. The proposed fiber laser with switchable CS and SP is attractive for ultrashort pulse generation and fast measurements in practical applications.
\end{abstract}

\section{Keywords}

Ultrashort Pulse Generation, Fiber Laser, Mode Locking, Solitons

\section{Introduction}

Due to their excellent features of generating ultra-short pulses, passively modelocked fiber lasers have drawn a lot of attention [1] [2] [3] [4]. Ultrafast fiber lasers with the merits of excellent heat dissipation [5] [6] [7], freedom from alignment [8] [9] and compact laser cavity design [10] [11] are widely applied in the fields of biomedical diagnostics [12], optical communications [12] [13] [14] and nonlinear optics [13] [15]. Passively mode-locked fiber lasers can be obtained by different types of nonlinear elements, such as nonlinear polarization 
rotation (NPR) [16] [17] [18] [19], semiconductor saturable absorber mirrors [11] [20] [21], nonlinear optical loop mirrors [22] [23] [24], graphene [25] [26], graphite nano-particles [27] and single-wall carbon nanotubes (SWNTs) [28] [30]. Among them, SWNTs can work as excellent saturable absorbers (SAs) due to their advantages of fast recovery time, high damage threshold, good modulation depth, broad operation bandwidth, outstand environmental stability, and affordable fabrication [31] [32] [33]. There are many investigators that pay great attention to SWNTs as SAs. A fiber laser generating a $74 \mathrm{fs}$ pulse with $63 \mathrm{~nm}$ spectral width based on nanotube mode locker have been proposed by Popa et al. [34]. Recently, a distributed ultrafast laser was proposed firstly by Liu et al. by means of the linearly chirped grating technique [40]. Moreover, broadband operation which is the superior feature of SWNTs can be realized by mixing SWNTs with different diameters, because the diameter and chirality of nanotubes determine the absorption peak of SWNTs [35].

A long interaction length for the guided light and the nanotubes is introduced by the D-shaped fiber saturable absorber, which guarantees an efficient nonlinear effect so that it facilitates laser mode locking even though the laser is under various conditions [36]. Higher intracavity power can be introduced for higher energy pulse formation, because only a part of the optical power of the propagating mode interacts with the SWNTs for mode locking. Lots of researchers have focused on evanescent-field interaction with SWNTs due to the outstanding performance of higher energy pulse formation and low CNT-density threshold [37]. The first experimental observation of four-wave mixing in SWNTs deposited on a DF has been reported by Chow et al. [38]. By using the evanescent-field interaction of propagating light with the nanotubes, a mode locker that is immunized to the high optical power induced damage have been demonstrated by Song et al. [36] [39].

Different types of mode-locked pulses have been obtained due to different designs of fiber laser cavity, such as CS [29] [40] [41], SP [42], self-similar pulse [43] [44], and dissipative soliton (DS) [5] [17] [45]. Because of the interaction between the fiber anomalous dispersion and nonlinear optical Kerr effect, CSs can be formed in negative dispersive regime [5] [41] [46]. When the frequencies of dispersive waves are phase-matched, discrete sidebands whose positions are determined by the cavity length, net dispersion, and pulse duration will generate and distribute on both sides of the spectrum [46] [47] [48]. When the net cavity dispersion approaches zero, SPs propagating in such a cavity are stretched and compressed periodically [21] [43] [49] [50]. Self-similar pluses operating in normal-dispersion regime are the parabolic pulses with self-similar evolution [43] [51] [52]. Moreover, the linear-chirped self-similar pulses can avoid pulse breaking when they propagate in the routes [53]. The multiple interactions between fiber nonlinearity, normal dispersion, saturable absorption effect, and spectral filtering effect result in the formation of DS [54]. DS has higher energy and wilder pulse width than that of CS, and its characteristics change dramati- 
cally when it travels along the laser cavity [55].

Although as a lot of progress has been made, fiber laser on many key physical parameters (such as pulse energy, pulse width and peak power, etc.) still lags behind that of the solid laser. Therefore, development of ultrashort pulse fiber laser has important practical significance and broad market prospect. In this paper, we propose a fiber laser that can deliver switchable CS and SP based on a SWNT mode-locker deposited on D-shaped fiber (DF). The proposed fiber laser can generate SP or CS with central wavelength of $1530 \mathrm{~nm}$ by tuning pump power and polarization controller (PC) properly. The spectral bandwidth and pulse duration of the CS are $4.46 \mathrm{~nm}$ and $\sim 0.7 \mathrm{ps}$, and these of the SP are $9.59 \mathrm{~nm}$ and $\sim 1.0$ ps. A NPR-induced filter is formed by the polarization sensitive DF-SA and PC. The stretching factor plays a key role on the switchable mode-locking operation, which can be tuned by the NPR-induced filter. The saturable absorber (SA) can withstand pump power up to $550 \mathrm{~mW}$ and deliver stable SP or CS without damage. It is attractive for practical applications of ultrashort pulse generation and fast measurements to implement our fiber laser with switchable CS and SP.

\section{Experimental Setup}

Figure 1 shows the schematic diagram of our experimental setup. In the fiber laser cavity, a $980 \mathrm{~nm}$ laser diode (LD) is used as pump source via a $980 / 1550 \mathrm{~nm}$ wavelength-division multiplexer (WDM) coupler; an 18-m erbium-doped fiber (EDF) with the group velocity dispersion (GVD) parameter of $-9 \mathrm{ps} / \mathrm{nm} / \mathrm{km}$ and the absorption of $3 \mathrm{~dB} / \mathrm{m}$ at $980 \mathrm{~nm}$ acts as the gain media; a PC in the cavity is used to adjust the state of polarization; the $10 \%$ port of a $90 / 10$ coupler is adopted to extract the laser for measurement; the DF-SA integrated into the

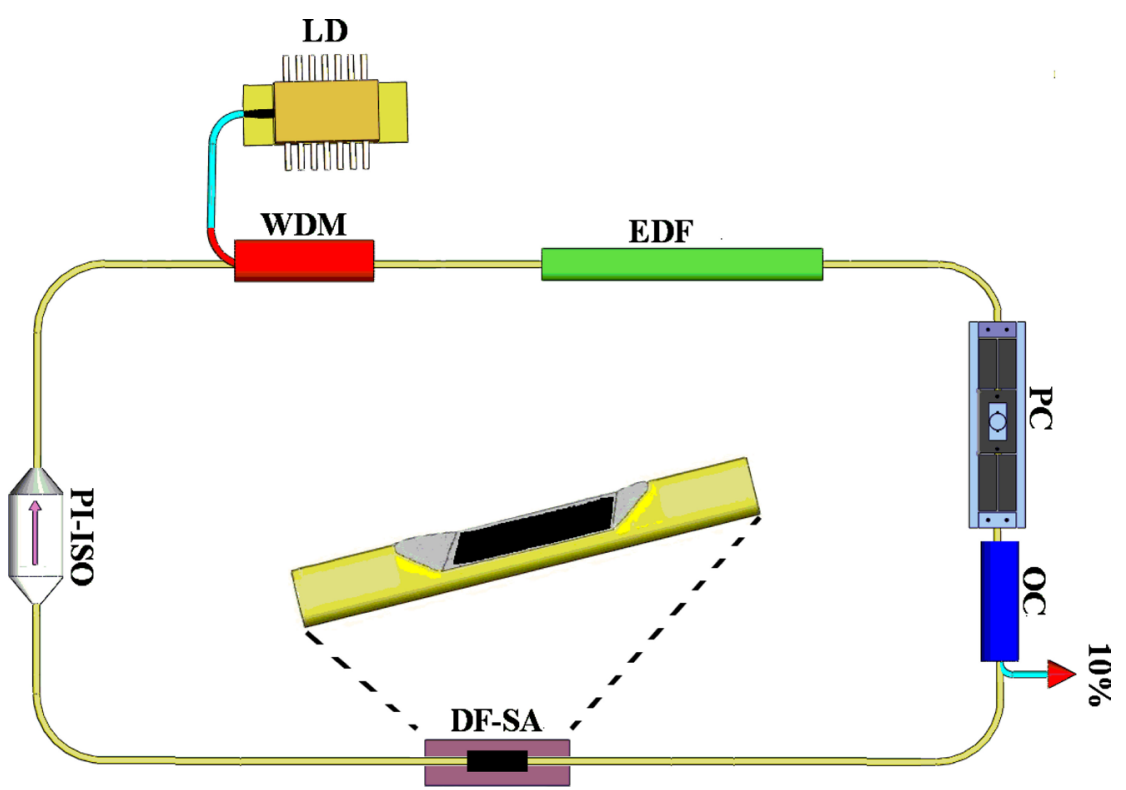

Figure 1. Schematic diagram of the laser system. LD, laser diode; WDM, wavelength-division multiplexer; EDF, erbium-doped fiber; PC, polarization controller; DF-SA, D-shaped-fiber saturable absorber; PI-ISO, polarization-insensitive isolator. 
cavity is made by using an optical-deposition technique to deposit SWNTs on the DF [56]; a polarization independent isolator (PI-ISO) is adopted to realize unidirectional operation. And all the rest of fibers are single mode fibers (SMFs) whose dispersion parameter is $17 \mathrm{ps} / \mathrm{nm} / \mathrm{km}$. The devices of a commercial autocorrelator (AC), a radio frequency ( $\mathrm{RF}$ ) analyzer, a digital storage oscilloscope, and an optical spectrum analyzer (OSA) are employed to monitor the laser outputs simultaneously.

The employed DF is fabricated by side-polishing SMFs [57] [58]. The whole procedure is monitored by a power meter to estimate the distance from polished surface to the fiber core. The DF-SA is made by using an optical-deposition technique to deposit SWNTs on the DF [45] and the insertion loss of the DF-SA is measured as $5 \mathrm{~dB}$. As shown in Figure 1, the propagating light follows the route as WDM $\rightarrow \mathrm{EDF} \rightarrow \mathrm{PC} \rightarrow \mathrm{OC} \rightarrow \mathrm{DF}-\mathrm{SA} \rightarrow \mathrm{PI}-\mathrm{ISO} \rightarrow \mathrm{WDM}$. The dispersion parameters $D$ of EDF and SMF are about -9 and $17 \mathrm{ps} /(\mathrm{nm} \cdot \mathrm{km})$. The net dispersion and the cavity length are $-0.045 \mathrm{ps}^{2}$ and $\sim 29.6 \mathrm{~m}$, respectively. By tuning the polarization state of propagating light through adjusting PC and providing different pump power appropriately, we can realize switchable SP and CS.

\section{Results and Discussions}

With the pump power of $\sim 50 \mathrm{~mW}$, the continuous wave (CW) is observed from the outputted fiber laser. When the total pump power is increased to $\sim 70 \mathrm{~mW}$, CS with its typical spectrum is shown in Figure 2(a). Figure 2(a) shows the output spectrum of CS with a bandwidth of $4.46 \mathrm{~nm}$. It can be seen that obvious Kelly sidebands discretely distribute on both sides of spectrum, which reflects the soliton features of the pulses [28] [37] [59]. The sidebands originate from the constructive interference between the soliton and dispersive waves [26] [59]. The
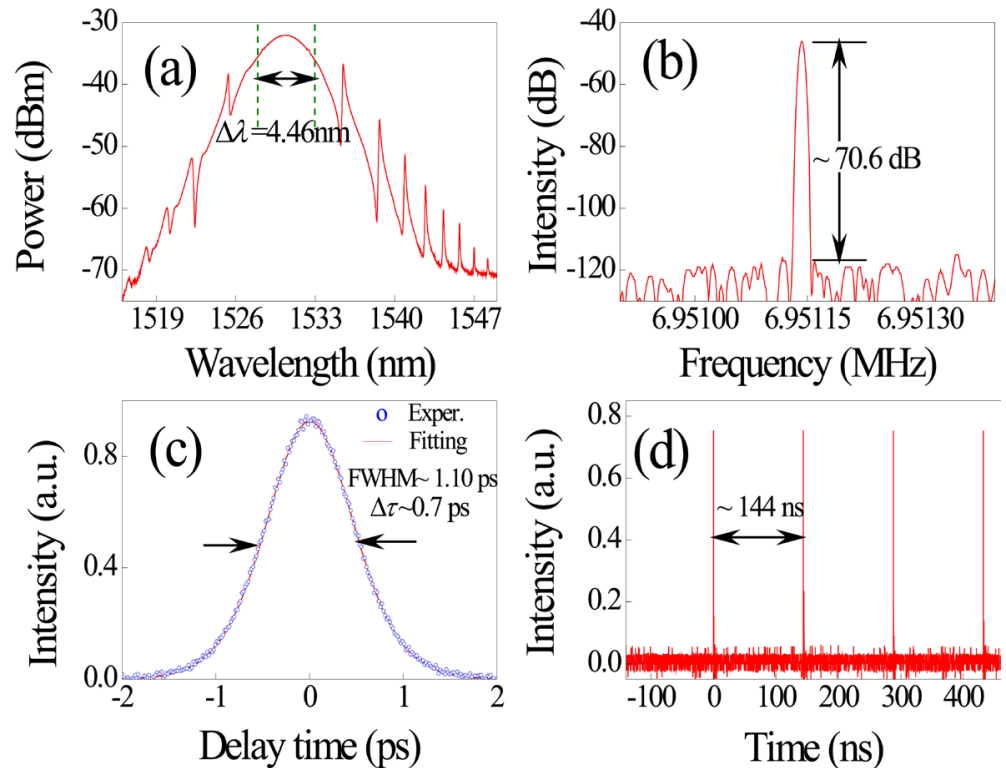

Figure 2. Conventional soliton (CS) operation of (a) Optical spectra, (b) Radio frequency spectrum (RF) spectra, (c) Autocorrelation (AC) trace, (d) Oscilloscope trace. 
central wavelength of the CS locates at $\sim 1530.6 \mathrm{~nm}$, which is mainly determined by the diameter distribution of nanotubes in SWNT-SA, the intracavity loss, the transmittance peak of birefringence-induced filter, and the gain profile of EDF [42] [60]. In Figure 2(b), the RF spectrum with the range of $1 \mathrm{KHz}$ shows that the fundamental repetition rate of CS is $\sim 6.95 \mathrm{MHz}$, corresponding to a roundtrip time of $\sim 144$ ns. Our experimental setup of the nanotube-based Fiber Laser can realize the ultrashort pulse generation and fast measurements. The signalto-noise ratio (SNR) is $\sim 70.6 \mathrm{~dB}$, which confirms that the CS operates at the stable mode-locking state. As shown in Figure 2(c), the full width at half maximum (FWHM) is $1.10 \mathrm{ps}$, and the pulse duration is estimated as 0.7 ps by using a $\mathrm{Sech}^{2}$ fit. The time bandwidth product (TBP) is calculated as 0.39 , indicating that the output CS is slightly chirped. The oscilloscope trace plotted in Figure 2(d) demonstrates that the pulse-to-pulse separation is about $144 \mathrm{~ns}$, corresponding to the cavity length of $\sim 29.6 \mathrm{~m}$.

When the output power is reduced to half of the original power, frequency bandwidth is 3-dB bandwidth. With increasing the pump power to $\sim 90 \mathrm{~mW}$ and optimizing the PC, the typical spectrum of SP is shown in Figure 3(a). The SP centered at $\sim 1530.3 \mathrm{~nm}$ exhibits a smooth spectral profile with a 3-dB bandwidth of $\sim 9.59 \mathrm{~nm}$. Figure 3 (b) shows the RF spectrum of SP revealing that fundamental repetition rate is $\sim 6.95 \mathrm{MHz}$, which is almost the same to that of CS due to the similar central wavelength. Meanwhile, we can get the SNR of $\sim 52 \mathrm{~dB}$, which indicates the stability of the SP. The corresponding autocorrelation trace is depicted in Figure $3(\mathrm{c})$. The FWHM of SP is $\sim 1.42 \mathrm{ps}$, and the pulse duration is estimated as 1.0 ps if a Gaussian fits the curve. Therefore, the TBP of SP is calculated as 1.2 which is much larger than the transform limit, and is the typical characteristic of SP. Figure 3(d) illustrates the oscilloscope trace, showing that
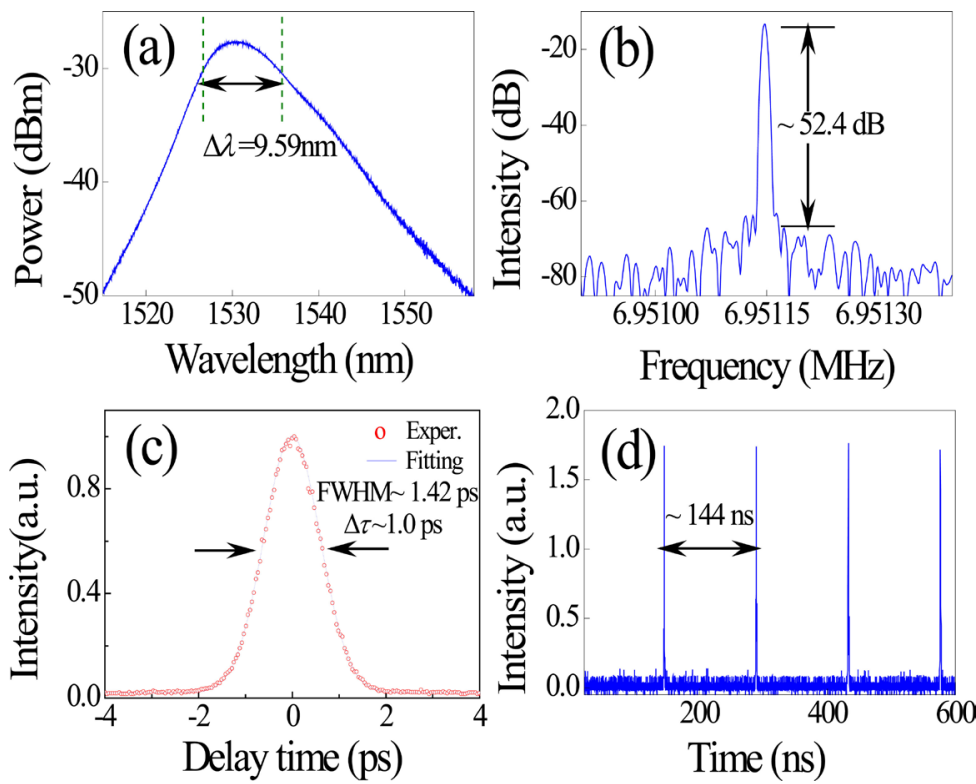

Figure 3. Stretch pulse (SP) operation of (a) Optical spectra, (b) RF spectra, (c) AC trace, (d) Oscilloscope trace. 
the round-trip time of the cavity is $\sim 144 \mathrm{~ns}$.

In our experiment, the spectral filtering effect plays an important role on the pulse formation. The polarization sensitive DF-SA and PC can be considered as a NPR-induced spectral filter and the intensity transmission of light through the filter, $T$, can be expressed as [19]

$$
T=\sin ^{2}(\theta) \sin ^{2}(\varphi)+\cos ^{2}(\theta) \cos ^{2}(\varphi)+0.5 \sin (2 \theta) \sin (2 \varphi) \cos \left(\phi_{1}+\phi_{2}\right)
$$

where $\phi_{1}$ is the phase delay caused by the PC and $\phi_{2}$ is the phase delay resulting from the fiber including both the linear phase delay and the nonlinear phase delay. The polarizer and analyzer have an orientation of angles $\theta$ and $\varphi$ with respect to the fast axis of the fiber, respectively. From Equation (1), one knows that the bandwidth of NPR-induced filter varies with PC states. Therefore, the different PC' settings will generate pulses with different bandwidths, which in turn lead to various stretching factors [52]. When the stretching factor is small enough and the interplay between anomalous cavity dispersion and positive Kerr nonlinearity of the fiber is balanced, the laser will operate in the soliton regime with spectral sidebands. As the stretching factor increases, spectral sideband generation will be reduced and there will be less dispersive radiation between pulses. Thus, stretched pulses will be generated with cleaner and broader spectra in the cavity when the stretching factor is large enough. Moreover, the solitons can be maintained as the pump power changes from the self-starting threshold to the maximum available power $(550 \mathrm{~mW})$, so the SA displays an ultra-high optical damage threshold.

\section{Conclusion}

By employing a DF-SA, we have proposed a simple and compact fiber laser system that can deliver CS and SP. With optimizing the PC properly and adjusting the pump power appropriately, we can achieve the CS and SP that have the similar central wavelengths and fundamental repetition rates. Because of the different pump powers and operations on PC, the stretching factor which is tunable due to the spectral filtering effect induced by NPR varied dramatically, they have different pulse durations, 3-dB bandwidths and optical spectra. The pulse duration and 3-dB bandwidth of CS centered at $1530.6 \mathrm{~nm}$ are $\sim 0.7 \mathrm{ps}$ and $4.46 \mathrm{~nm}$ while that of SP with central wavelength of $1530.3 \mathrm{~nm}$ are $\sim 1.0 \mathrm{ps}$ and $9.59 \mathrm{~nm}$, respectively. Our experimental setup of the nanotube-based Fiber Laser can realize the ultrashort pulse generation and fast measurements. But the key physical parameters (such as pulse energy, pulse width and peak power) still lag behind that of the solid laser. It is attractive and convenient for practical research applications of ultrashort pulse field because of the proposed fiber laser delivering two types of pulses.

\section{Acknowledgements}

The work was supported in part by national natural science foundation of China (No. 61540012), National scholarship foundation (201609480004), Natural Science 
Foundation of Hunan Province (2017JJ2097, 2018JJ2132), and Education Department of Hunan Province (16A067), Hunan University of Science and Technology Research Fund (No. 908A11567), and Science research fund of Hunan Province Key Laboratory of Coal Resources Clean-Utilization and Mine Environment Protection (No. E21698).

\section{References}

[1] Liu, X.M., Yang, H.R., Cui, Y.D., Chen, G.W., Yang, Y., Wu, X.Q. and Guo, J. (2016) Graphene-Clad Microfibre Saturable Absorber for Ultrafast Fibre Lasers. Scientific Reports, 6, 26024. https://doi.org/10.1038/srep26024

[2] Cui, Y., Lu, F. and Liu, X. (2016) MoS2-Clad Microfibre Laser Delivering Conventional, Dispersion-managed and Dissipative Solitons. Scientific Reports, 6, 30524. https://doi.org/10.1038/srep30524

[3] Grelu, P. and Akhmediev, N. (2012) Dissipative Solitons for Mode-Locked Lasers. Nature Photonics, 6, 84-92. https://doi.org/10.1038/nphoton.2011.345

[4] Fermann, M.E. and Hartl, I. (2009) Ultrafast Fiber Laser Technology. IEEE Journal of Selected Topics in Quantum Electronics, 15, 191-206.

https://doi.org/10.1109/JSTQE.2008.2010246

[5] Duan, L., Liu, X., Mao, D., Wang, L. and Wang, G. (2012) Experimental Observation of Dissipative Soliton Resonance in an Anomalous-Dispersion Fiber Laser. $O p$ tics Express, 20, 265-270. https://doi.org/10.1364/OE.20.000265

[6] Wang, L.R., Liu, X.M. and Gong, Y.K. (2009) Giant-Chirp Oscillator for Ultra-Large Net-Normal-Dispersion Fiber Lasers. Laser Physics Letters, 7, 63-67.

https://doi.org/10.1002/lapl.200910109

[7] Kobtsev, S., Kukarin, S., Smirnov, S., Turitsyn, S. and Latkin, A. (2009) Generation of Double-Scale Femto/Pico-Second Optical Lumps in Mode-Locked Fiber Lasers. Optics Express, 17, 20707-20713. https://doi.org/10.1364/OE.17.020707

[8] Liu, X.M., Han, X.X. and Yao, X.K. (2016) Discrete Bisoliton Fiber Laser. Scientific Reports, 6, 34414. https://doi.org/10.1038/srep34414

[9] Sun, H.B., Liu, X.M., Gong, Y.K., Li, X.H. and Wang, L.R. (2010) Broadly Tunable Dual-Wavelength Erbium-Doped Ring Fiber Laser Based on a High-Birefringence Fiber Loop Mirror. Laser Physics, 20, 522-527. https://doi.org/10.1134/S1054660X10030175

[10] Li, X.H., Liu, X.M., Gong, Y.K., Sun, H.B., Wang, L.R. and Lu, K.Q. (2009) A Novel Erbium/Ytterbium Co-Doped Distributed Feedback Fiber Laser with Single-Polarization and Unidirectional Output. Laser Physics Letters, 7, 55-59. https://doi.org/10.1002/lapl.200910100

[11] Keller, U. (2003) Recent Developments in Compact Ultrafast Lasers. Nature, 424, 831-838. https://doi.org/10.1038/nature01938

[12] Wise, F.W., Chong, A. and Renninger, W.H. (2008) High-Energy Femtosecond Fiber Lasers Based on Pulse Propagation at Normal Dispersion. Laser \& Photonics Reviews, 2, 58-73. https://doi.org/10.1002/lpor.200710041

[13] Han, D.D., Liu, X.M., Cui, Y.D., Wang, G.X., Zeng, C. and Yun, L. (2014) Simultaneous Picosecond and Femtosecond Solitons Delivered from a Nanotube-Mode-Locked All-Fiber Laser. Optics Letters, 39, 1565-1568. https://doi.org/10.1364/OL.39.001565

[14] Liu, X.M., Chung, Y., Lin, A., Zhao, W., Lu, K.Q., Wang, Y.S. and Zhang, T.Y. 
(2008) Tunable and Switchable Multi-Wavelength Erbium-Doped Fiber Laser with Highly Nonlinear Photonic Crystal Fiber and Polarization Controllers. Laser Physics Letters, 5, 904-907. https://doi.org/10.1002/lapl.200810085

[15] Cui, Y.D., Liu, X.M. and Zeng, C. (2014) Conventional and Dissipative Solitons in a CFBG-Based Fiber Laser Mode-Locked with a Graphene-Nanotube Mixture. Laser Physics Letters, 11, Article ID: 055106. https://doi.org/10.1088/1612-2011/11/5/055106

[16] Luo, Z.C., Luo, A.P., Xu, W.C., Song, C.X., Gao, Y.X. and Chen, W.C. (2009) Sideband Controllable Soliton All-Fiber Ring Laser Passively Mode-Locked by Nonlinear Polarization Rotation. Laser Physics Letters, 6, 582-585. https://doi.org/10.1002/lapl.200910032

[17] Liu, X. (2009) Dissipative Soliton Evolution in Ultra-Large Normal-Cavity Dispersion Fiber Lasers. Optics Express, 17, 9549-9557. https://doi.org/10.1364/OE.17.009549

[18] Wang, W., Meng, H.Y., Wu, X.W., Xiong, R., Xue, H.C., Tan, C.H. and Huang, X.G. (2012) A Nonlinear Polarization Rotation-Based Linear Cavity Waveband Switchable Multi-Wavelength Fiber Laser. Laser Physics Letters, 10, Article ID: 015104.

[19] Liu, X. (2010) Hysteresis Phenomena and Multipulse Formation of a Dissipative System in a Passively Mode-Locked Fiber Laser. Physical Review A, 81, Article ID: 023811. https://doi.org/10.1103/PhysRevA.81.023811

[20] Ouyang, C., Shum, P., Wu, K., Wong, J.H., Lam, H.Q. and Aditya, S. (2011) Bidirectional Passively Mode-Locked Soliton Fiber Laser with a Four-Port Circulator. Optics Letters, 36, 2089-2091. https://doi.org/10.1364/OL.36.002089

[21] Liu, X. and Cui, Y. (2015) Flexible Pulse-Controlled Fiber Laser. Scientific Reports, 5, Article No. 9399. https://doi.org/10.1038/srep09399

[22] Xu, B., Martinez, A., Set, S.Y., Goh, C.S. and Yamashita, S. (2013) A Net Normal Dispersion All-Fiber Laser Using a Hybrid Mode-Locking Mechanism. Laser Physics Letters, 11, Article ID: 025101.

[23] Yun, L. and Liu, X. (2012) Generation and Propagation of Bound-State Pulses in a Passively Mode-Locked Figure-Eight Laser. IEEE Photonics Journal, 4, 512-519. https://doi.org/10.1109/JPHOT.2012.2191948

[24] Salhi, M., Haboucha, A., Leblond, H. and Sanchez, F. (2008) Theoretical Study of Figure-Eight All-Fiber Laser. Physical Review A, 77, Article ID: 033828. https://doi.org/10.1103/PhysRevA.77.033828

[25] Bonaccorso, F., Sun, Z., Hasan, T. and Ferrari, A.C. (2010) Graphene Photonics and Optoelectronics. Nature Photonics, 4, 611-622. https://doi.org/10.1038/nphoton.2010.186

[26] Cui, Y. and Liu, X. (2013) Graphene and Nanotube Mode-Locked Fiber Laser Emitting Dissipative and Conventional Solitons. Optics Express, 21, 18969-18974. https://doi.org/10.1364/OE.21.018969

[27] Lin, Y.H. and Lin, G.R. (2012) Free-Standing Nano-Scale Graphite Saturable Absorber for Passively Mode-Locked Erbium Doped Fiber Ring Laser. Laser Physics Letters, 9, 398-404. https://doi.org/10.7452/lapl.201210010

[28] Zeng, C., Liu, X. and Yun, L. (2013) Bidirectional Fiber Soliton Laser Mode-Locked by Single-Wall Carbon Nanotubes. Optics Express, 21, 18937-18942. https://doi.org/10.1364/OE.21.018937

[29] Liu, X., Han, D., Sun, Z., Zeng, C., Lu, H., Mao, D. and Wang, F. (2013) Versatile Multi-Wavelength Ultrafast Fiber Laser Mode-Locked by Carbon Nanotubes. 
Scientific Reports, 3, Article No. 2718. https://doi.org/10.1038/srep02718

[30] Im, J.H., Choi, S.Y., Rotermund, F. and Yeom, D.I. (2010) All-Fiber Er-Doped Dissipative Soliton Laser Based on Evanescent Field Interaction with Carbon Nanotube Saturable Absorber. Optics Express, 18, 22141-22146. https://doi.org/10.1364/OE.18.022141

[31] Set, S.Y., Yaguchi, H., Tanaka, Y. and Jablonski, M. (2004) Ultrafast Fiber Pulsed Lasers Incorporating Carbon Nanotubes. IEEE Journal of Selected Topics in Quantum Electronics, 10, 137-146. https://doi.org/10.1109/JSTQE.2003.822912

[32] Wang, Q., Chen, T., Li, M., Zhang, B., Lu, Y. and Chen, K.P. (2013) All-Fiber Ultrafast Thulium-Doped Fiber Ring Laser with Dissipative Soliton and Noise-Like Output in Normal Dispersion by Single-Wall Carbon Nanotubes. Applied Physics Letters, 103, Article ID: 011103.

[33] Scardaci, V., Sun, Z., Wang, F., Rozhin, A.G., Hasan, T., Hennrich, F. and Ferrari, A.C. (2008) Carbon Nanotube Polycarbonate Composites for Ultrafast Lasers. Advanced Materials, 20, 4040-4043. https://doi.org/10.1002/adma.200800935

[34] Popa, D., Sun, Z., Hasan, T., Cho, W.B., Wang, F., Torrisi, F. and Ferrari, A.C. (2012) 74-fs Nanotube-Mode-Locked Fiber Laser. Applied Physics Letters, 101, Article ID: 153107. https://doi.org/10.1063/1.4757293

[35] Kataura, H., Kumazawa, Y., Maniwa, Y., Umezu, I., Suzuki, S., Ohtsuka, Y. and Achiba, Y. (1999) Optical Properties of Single-Wall Carbon Nanotubes. Synthetic Metals, 103, 2555-2558. https://doi.org/10.1016/S0379-6779(98)00278-1

[36] Song, Y.W., Yamashita, S. and Maruyama, S. (2008) Single-Walled Carbon Nanotubes for High-Energy Optical Pulse Formation. Applied Physics Letters, 92, Article ID: 021115 .

[37] Song, Y.W., Yamashita, S., Goh, C.S. and Set, S.Y. (2007) Carbon Nanotube Mode Lockers with Enhanced Nonlinearity via Evanescent Field Interaction in D-Shaped Fibers. Optics Letters, 32, 148-150. https://doi.org/10.1364/OL.32.000148

[38] Chow, K.K. and Yamashita, S. (2009) Four-Wave Mixing in a Single-Walled Carbon-Nanotube-Deposited D-Shaped Fiber and Its Application in Tunable Wavelength Conversion. Optics Express, 17, 15608-15613. https://doi.org/10.1364/OE.17.015608

[39] Song, Y.W., Yamashita, S., Einarsson, E. and Maruyama, S. (2007) All-Fiber Pulsed Lasers Passively Mode Locked by Transferable Vertically Aligned Carbon Nanotube Film. Optics Letters, 32, 1399-1401. https://doi.org/10.1364/OL.32.001399

[40] Liu, X., Cui, Y., Han, D., Yao, X. and Sun, Z. (2015) Distributed Ultrafast Fibre Laser. Scientific Reports, 5, Article No. 12731.

[41] Liu, X. (2011) Soliton Formation and Evolution in Passively-Mode-Locked Lasers with Ultralong Anomalous-Dispersion Fibers. Physical Review A, 84, Article ID: 023835. https://doi.org/10.1103/PhysRevA.84.023835

[42] Nishizawa, N., Nozaki, Y., Itoga, E., Kataura, H. and Sakakibara, Y. (2011) Dispersion-Managed, High-Power, Er-Doped Ultrashort-Pulse Fiber Laser Using Carbon-Nanotube Polyimide Film. Optics Express, 19, 21874-21879.

https://doi.org/10.1364/OE.19.021874

[43] Ilday, F.Ö., Buckley, J.R., Clark, W.G. and Wise, F.W. (2004) Self-Similar Evolution of Parabolic Pulses in a Laser. Physical Review Letters, 92, Article ID: 213902. https://doi.org/10.1364/NLGW.2004.MD8

[44] Dudley, J.M., Finot, C., Richardson, D.J. and Millot, G. (2007) Self-Similarity in Ultrafast Nonlinear Optics. Nature Physics, 3, 597-603. 
https://doi.org/10.1038/nphys705

[45] Mao, D., Liu, X.M., Wang, L.R., Hu, X.H. and Lu, H. (2010) Partially Polarized Wave-Breaking-Free Dissipative Soliton with Super-Broad Spectrum in a ModeLocked Fiber Laser. Laser Physics Letters, 8, 134-138. https://doi.org/10.1002/lapl.201010105

[46] Han, D. and Liu, X. (2012) Sideband-Controllable Mode-Locking Fiber Laser Based on Chirped Fiber Bragg Gratings. Optics Express, 20, 27045-27050.

https://doi.org/10.1364/OE.20.027045

[47] Lin, Y.H., Yang, C.Y., Liou, J.H., Yu, C.P. and Lin, G.R. (2013) Using Graphene Nano-Particle Embedded in Photonic Crystal Fiber for Evanescent Wave ModeLocking of Fiber Laser. Optics Express, 21, 16763-16776. https://doi.org/10.1364/OE.21.016763

[48] Lin, Y.H. and Lin, G.R. (2013) Kelly Sideband Variation and Self Four-Wave-Mixing in Femtosecond Fiber Soliton Laser Mode-Locked by Multiple Exfoliated Graphite Nano-Particles. Laser Physics Letters, 10, Article ID: 045109. https://doi.org/10.1088/1612-2011/10/4/045109

[49] Yu, T., Golovchenko, E.A., Pilipetskii, A.N. and Menyuk, C.R. (1997) Dispersion-Managed Soliton Interactions in Optical Fibers. Optics Letters, 22, 793-795. https://doi.org/10.1364/OL.22.000793

[50] Salhi, M., Leblond, H. and Sanchez, F. (2003) Theoretical Study of the StretchedPulse Erbium-Doped Fiber Laser. Physical Review A, 68, Article ID: 033815. https://doi.org/10.1103/PhysRevA.68.033815

[51] Bale, B.G. and Wabnitz, S. (2010) Strong Spectral Filtering for a Mode-Locked Similariton Fiber Laser. Optics Letters, 35, 2466-2468. https://doi.org/10.1364/OL.35.002466

[52] Oktem, B., Ülgüdür, C. and Ilday, F.Ö. (2010) Soliton-Similariton Fibre Laser. Nature Photonics, 4, 307-311. https://doi.org/10.1038/nphoton.2010.33

[53] Anderson, D., Desaix, M., Karlsson, M., Lisak, M. and Quiroga-Teixeiro, M.L. (1993) Wave-Breaking-Free Pulses in Nonlinear-Optical Fibers. JOSA B, 10, 1185 1190. https://doi.org/10.1364/JOSAB.10.001185

[54] Zhao, L.M., Lu, C., Tam, H.Y., Wai, P.K.A. and Tang, D.Y. (2009) Gain Dispersion for Dissipative Soliton Generation in All-Normal-Dispersion Fiber Lasers. Applied Optics, 48, 5131-5137. https://doi.org/10.1364/AO.48.005131

[55] Liu, X.M. and Mao, D. (2010) Compact All-Fiber High-Energy Fiber Laser with Sub-300-fs Duration. Optics Express, 18, 8847-8852. https://doi.org/10.1364/OE.18.008847

[56] Kashiwagi, K. and Yamashita, S. (2009) Deposition of Carbon Nanotubes around Microfiber via Evanascent Light. Optics Express, 17, 18364-18370. https://doi.org/10.1364/OE.17.018364

[57] Tseng, S.M. and Chen, C.L. (1992) Side-Polished Fibers. Applied Optics, 31, 3438-3447. https://doi.org/10.1364/AO.31.003438

[58] Ma, S.P. and Tseng, S.M. (1997) High-Performance Side-Polished Fibers and Applications as Liquid Crystal Clad Fiber Polarizers. Journal of Lightwave Technology, 15, 1554-1558. https://doi.org/10.1109/50.618389

[59] Nelson, L.E., Jones, D.J., Tamura, K., Haus, H.A. and Ippen, E.P. (1997) Ultrashort-Pulse Fiber Ring Lasers. Applied Physics B: Lasers and Optics, 65, 277-294. https://doi.org/10.1007/s003400050273 
[60] Tran, T.V.A., Lee, K., Lee, S.B. and Han, Y.G. (2008) Switchable Multiwavelength Erbium Doped Fiber Laser Based on a Nonlinear Optical Loop Mirror Incorporating Multiple Fiber Bragg Gratings. Optics Express, 16, 1460-1465.

https://doi.org/10.1364/OE.16.001460 